Natalie Binczek · Cornelia Epping-Jäger (Hg.)

Das Hörbuch 
Natalie Binczek · Cornelia Epping-Jäger (Hg.)

\title{
Das Hörbuch
}

Praktiken audioliteralen Schreibens und Verstehens

\author{
Wilhelm Fink
}


Diese Publikation entstand im Rahmen des von der DFG geförderten Forschungsprojektes „Poetik und Hermeneutik des Hörbuchs: akustische Texte in der deutschsprachigen Gegenwartsliteratur". Sie wurde mithilfe von der DFG und dem Rektor der Ruhr- Universität Bochum zur Verfügung gestellten Mitteln gedruckt.

Umschlagabbildung:

White Ear (C) 3dbobber-Fotolia.com

Bibliografische Information der Deutschen Nationalbibliothek

Die Deutsche Nationalbibliothek verzeichnet diese Publikation in der Deutschen Nationalbibliografie; detaillierte bibliografische Daten sind im Internet über http://dnb.d-nb.de abrufbar.

Alle Rechte, auch die des auszugsweisen Nachdrucks, der fotomechanischen Wiedergabe und der Übersetzung, vorbehalten. Dies betrifft auch die Vervielfältigung und Übertragung einzelner

Textabschnitte, Zeichnungen oder Bilder durch alle Verfahren wie Speicherung und Übertragung auf Papier, Transparente, Filme, Bänder, Platten und andere Medien, soweit es nicht $\$ \$ 53$ und 54 UrhG ausdrücklich gestatten.

(C) 2014 Wilhelm Fink Verlag, München

(Wilhelm Fink GmbH \& Co. Verlags-KG, Jühenplatz 1, D-33098 Paderborn)

Internet: www.fink.de

Einbandgestaltung: Evelyn Ziegler, München

Printed in Germany

Herstellung: Ferdinand Schöningh GmbH \& Co. KG, Paderborn

ISBN 978-3-7705-5346-4 


\section{INHALT}

NATAlie BinCZEK / CORNELIA EPPING-JÄGER

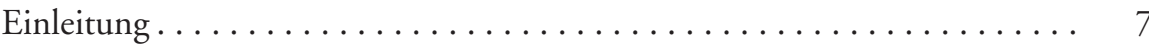

HARUN MAYE

Literatur aus der Sprechmaschine. Zur Mediengeschichte der

Dichterlesung von Klopstock bis Rilke

STEFFEN WALLACH

Vor der Phonographie - Herders, Arnims und Brentanos volkspoetische

Gramma-Phonie .

AXEL VOLMAR

In Stahlgewittern. Mediale Rekonstruktionen der Klanglandschaft

des Ersten Weltkriegs in der Weimarer Republik ..................

THOMAS WeGMANN

Text-Akustik: Rundfunk und skripturale Oralität im Spätwerk

Gottfried Benns.

MANFRED SCHNEIDER

Lyrik im Zeitalter des digitalen Ohrschnullers ...

HeINZ Hiebler

Problemfeld ,Hörbuch'. Das Hörbuch in der medienorientierten

Literaturwissenschaft.

ARMIN SCHÄFER

Unterwegs zur akustischen Literatur: Karl Kraus ..

CORNELIA EPPING-JÄGER

,Die verfluchte Gegenwart - und dann das Erstaunen, dass ich das sage؛.

Rolf Dieter Brinkmann und das Tonband als produktionsästhetische

Maschine.

NATALIE BINCZEK

Einen Text ,zu umschneiden und von seiner Unterlage abzupräparieren`.

Elfriede Jelineks „Moosbrugger will nichts von sich wissen“. . . 
WOLFGANG HAGEN

„Wer Bücher hört, kann auch Klänge sehen.“

Bemerkungen zur Synästhesie des Hörbuchs

Till DeMBECK

Akustische Untote. Buch und Hören um 1900 und heute . . . . . . . . . . . . 193

UWE WIRTH

Akustische Paratextualität, akustische Paramedialität . . . . . . . . . . . 215

LUDWIG JäGER

Audioliteralität. Eine Skizze zur Transkriptivität des Hörbuchs

Abbildungsverzeichnis.......................... 255

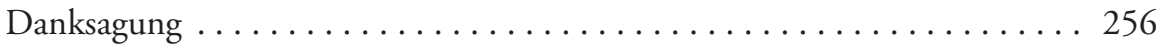

Autorinnen und Autoren. . . . . . . . . . . . . . . . . . 257 


\section{Till Dembeck}

\section{AKUSTISCHE UNTOTE BUCH UND HÖREN UM 1900 UND HEUTE}

Untote dienen häufig als Reflexionsfiguren für technische Medien und ihre Fähigkeit, das Leben der Vergangenheit zumindest in Teilen, gewissermaßen als Schatten, wiedererstehen zu lassen. Das gilt schon für die Schrift, von der immer wieder gesagt wurde, sie leiste eine gespenstische Vergegenwärtigung ,toter ' Rede; aber auch für den Film, der sich Figuren wie den Titelhelden aus Bram Stokers Dracula (1897) vielfach zu Nutze macht, um das eigene Schattenspiel mit einer wirkmächtigen Schattengestalt in Verbindung zu bringen. Der folgende Beitrag interessiert sich für eine Facette dieses Gesamtkomplexes, die für die Poetik des Hörbuchs eine zentrale Bedeutung hat, nämlich für das Untote als Reflexionsfigur phonographischer Medien, die bereits in Stokers Roman eine bestimmte Rolle spielen.

Die medienwissenschaftlichen loci classici für den Zusammenhang von Literatur und Phonographie sind bis heute Friedrich Kittlers Lektüre von Dracula und seine Anmerkungen zu Auguste Villiers de l'Isle-Adams L'Éve future (1886). ${ }^{1}$ Ich möchte Kittlers Impuls noch einmal aufnehmen - zum einen, um zu klären, aufgrund welcher spezifischen Struktureigenschaften das neue Analogmedium mit dem Untoten in Verbindung gebracht werden und so als defizitärer Wiedergänger der menschlichen Seele erscheinen kann; zum anderen, um frühe Konzepte gehörter/zu hörender Buchmedialität aufzudecken, wie sie noch in modernen Hörbüchern nachhallen, etwa in Georg Kleins Schlimme schlimme Medien von 2007. Dabei ist es notwendig, das Verhältnis von Textualität und Schallaufzeichnung präzise entlang der Unterscheidung digital/analog zu beschreiben, die in den einschlägigen Arbeiten zu Phonographie und Literatur immer noch zu wenig systematisch fruchtbar gemacht wird. Den Lektüren der Romane von Stoker (Abschnitt II) und Villiers de l'Isle-Adam (Abschnitt III) sowie des Hörbuchs von Klein (Abschnitt IV) werden daher einige allgemeinere Ausführungen zur Semantik der Phonographie am Ende des 19. Jahrhunderts vorgeschaltet.

1 Vgl. Friedrich Kittler: Draculas Vermächtnis, in: Ders.: Draculas Vermächtnis. Technische Schriften [1982], Leipzig 1993, S. 11-57; sowie Ders.: Aufschreibesysteme 1800 - 1900 [1985], München 1995, S. 441f., 449-452. 
Der Phonograph, wie ihn Thomas A. Edison 1877 erfand, war das erste Gerät, das Schallwellen nicht nur aufzeichnen, sondern auch reproduzieren konnte. Um die neue Medientechnologie, die sich von dieser Erfindung ausgehend entwickelte, rankte sich schnell eine umfassende Semantik. Im Folgenden möchte ich nur auf zwei Facetten dieser Semantik hinweisen, und zwar auf die Modellierung des Verhältnisses von Phonographie und Textualität einerseits und des Verhältnisses von Phonographie und menschlicher Seele andererseits; beides steht in einem engen Zusammenhang zueinander.

Edison schlägt gleich in den ersten Texten über seine Erfindung ihre Nutzung für die Produktion von Hörbüchern vor - und es ist von daher nur konsequent, wenn aktuelle Bestimmung des Hörbuchs bereits hier ansetzen. ${ }^{2}$ Es heißt etwa: „On four cylinders eight inches long, with a diameter of five, I can put the whole of ,Nicholas Nickleby' in phonogram form." Oder: „Authors [...] can also publish their novels of essays exclusively in phonograph form, so as to talk to their readers personally". ${ }^{3}$ Edison sah das Potential seiner Erfindung in erster Linie in der Textspeicherung - und nicht in der Musikdistribution, durch die das Verfahren später die größten unternehmerischen Erfolge erzielte. Er blieb damit ein Stück weit einem Paradigma verhaftet, das vor seiner Erfindung die Überlegungen zur Schallreproduktion beherrscht hatte. Über, Phonographie' hatte man bislang weniger hinsichtlich des Schalls im Allgemeinen nachgedacht als vielmehr hinsichtlich der menschlichen Stimme im Besonderen. ${ }^{4}$ Im Englischen bezeichnete der Terminus phonography vor Edison Verfahren der Stenographie; von Phonographie könnte man aber auch im Kontext der intensiven Bemühungen des 19. Jahrhunderts um phonetische Alphabete sprechen, also um Alphabete, die tatsächlich nicht nur Phoneme, sondern alle menschenmöglichen Phone verzeichnen können sollten. ${ }^{5}$ Auch

2 Vgl. Sandra Rühr: Tondokumente von der Walze zum Hörbuch: Geschichte - MedienspezitikRezeption, Göttingen 2008.

3 Thomas A. Edison: The Perfected Phonograph, in: The North American Review CXLVI (June 1888), S. 646 und 647; vgl. auch Anonym: Mr. Edisons Improved [!] Phonograph, in: Public Opinion IV (1887/8), S. $63 f$.

4 Vgl. hierzu und allgemein zum Verhältnis von Textualität und Phonographie vor und nach Edison: Lisa Gitelman: Scripts, Grooves, and Writing Machines. Representing Technology in the Edison Era, Stanford 1999.

5 Vgl. hierzu Till Dembeck: Phono-Graphie: Schallaufzeichnung und kultureller Vergleich 1800/1900, in: Markus Dauß/Ralf Haekel (Hg.): Leib/Seele - Geist/Buchstabe. Dualismen in der Ästhetik und den Künsten um 1800/1900, Würzburg 2007, S. 293-315. Einflussreiche Alphabete entwickelten etwa: Richard Carl Lepsius: Standard Alphabet for Reducing Unwritten Languages and Foreign Graphic Systems to a Uniform Orthography in European Letters [1855], London/Berlin 1863; F. H. du Bois-Reymond: Kadmus oder Allgemeine Alphabetik vom physikalischen, physiologischen und graphischen Stanpunkt, Berlin 1862; Alex. Melville Bell: Visible Speech: The Science of Universal Alphabetics; or self-interpreting physiological letters, for the writing of all languages in one alphabet. Illustrated by tables, diagrams, and examples, London/New York 1867. 
diese vor-Edisonsche Phonographie wollte das unfassliche Differenzierungspotential des menschenerzeugten Schalls dennoch technisch kontrollieren. Ihr bevorzugtes Mittel, um dieses Ziel zu erreichen, war die Steigerung und Ausdifferenzierung der verfügbaren Menge an diskreten Zeichen. Ihre Versuche, Schall wiederzugeben, verfuhren also digital; sie ging davon aus, dass das Wiederzugebende gewissermaßen schon zeichen- oder textförmig vorliege, bevor es als Schall reproduziert werden könne. Edison brach mit eben dieser Voraussetzung, konnte die Konsequenzen seiner Erfindung für das Verhältnis von Textualität und Phonographie aber nur zum Teil durchschauen.

Um diese Konsequenzen genauer zu erfassen, muss die Differenz zwischen analogen und digitalen Verfahren der Verzeichnung genauer beschrieben werden. Dabei ist die Einsicht entscheidend, dass für Digitalität die verlustfreie Kopierbarkeit der verwendeten Zeichen charakteristisch ist. ${ }^{6}$ Ein A, insofern es digitales Zeichen ist, bleibt ein A, auch wenn es beispielsweise in einer anderen Schriftart gedruckt wird. Dabei scheinen die Arten und Weisen, aus irgendwelchen ornamentalen Details ein neues A zu basteln, das dennoch als solches erkannt wird und dann, als digitales Zeichen, eben auch nur ein A ist, wenn auch nicht unbegrenzt, so doch grundsätzlich unvorhersehbar. ${ }^{7}$ Daraus lässt sich eine Differenz ableiten, die ich an anderer Stelle eingeführt habe, nämlich die Differenz zwischen Ornament und Figur. Diese Differenz ist für jede Digitalisierung konstitutiv: In diffusen und potentiell unbeschränkt differenzierten Sinnesdaten werden diskrete, wiedererkennbare Figuren markiert, die nur als solche verlustfrei kopierbar sind. ${ }^{8}$ An ihnen haftet Ornament, eine Fülle von Details, die für das Wiedererkennen der Figur nicht entscheidend sind, ohne die es sie allerdings auch nicht geben könnte;

6 Vgl. hierzu Till Dembeck: Phono-Graphie: Schallaufzeichnung und kultureller Vergleich 1800/1900 (Anm. 5), S. 304-307. Zu dieser Unterscheidung zwischen analoger und digitaler Differenzierung vgl. Anthony Wilden: System and Structure. Essays in Communication and Exchange [1972], London 1980, S. 169f.: „Analog differences are differences of magnitude, frequency, distribution, pattern, organization, and the like. Digital differences are those such as can be coded into DISTINCTIONS and OPPOSITIONS, and for this, there must be discrete elements with well-defined boundaries. [...] The discrete character of the signifier follows from its continuous substratum." Das bedeutet insbesondere, dass Digitalität ihrem Wesen nach nichts mit Elektronik zu tun hat, sondern sich die elektronische Datenverarbeitung umgekehrt der Digitalität bedient. Ein System, das 0 und 1 als einzige Zeichen benutzt, ist allerdings in der Tat das einfachste denkbare digitale System.

7 Vgl. Douglas R. Hofstadter: Metafont, Metamathematics, and Metaphysics: Comments on Donald Knuth's Article ,The Concept of a Meta-Font', in: Visible Language 16 (1982), S. 309-338.

8 Zur Unterscheidung zwischen Figur und Ornament Till Dembeck: Texte rahmen. Grenzregionen literarischer Werke im 18. Jahrhundert (Gottsched, Wieland, Moritz, Jean Paul), Berlin/New York 2007, S. 406-437; zu ihrer Anwendung auf Phonographie und Kultur vgl. ders.: Schibboleth/Sibboleth - Phonographie und kulturelle Kommunikation um 1900, in: Zeitschrift für Literaturwissenschaft und Linguistik 36/142 (2006), S. 43-68; und ders.: Phono-Graphie: Schallaufzeichnung und kultureller Vergleich 1800/1900 (Anm. 5.), S. 293 315. 
in diesem Ornament kann man dann zwar grundsätzlich wiederum Figuratives ausmachen und es auf diese Weise digitalisieren; durch diese Operation entsteht aber ein neues Ornamentales, woraus folgt, dass es eine vollständige Digitalisierung des Ornamentalen nicht gibt. Darin liegt letztlich das Scheitern der Phonographie vor Edison begründet, der es durchweg darum zu tun war, alle ornamentalen Möglichkeiten der menschlichen Stimme durch die Rationalisierung und Erweiterung des Figurenarsenals zu registrieren.

Gegenüber der digitalen Verzeichnung und Reproduktion des Schalls zeichnet sich die sogenannte, glyphische' Aufzeichnung, die zuerst Wilhelm Weber (für Stimmgabeln) und dann Leon Scott (für jede Art von Schall) erprobten und die auch Edisons Phonographie nutzt, dadurch aus, dass sie - aus der Sicht der Digitalität - ausschließlich Ornament aufzeichnet: Die Schallwellen selbst werden auf einer bewegten Unterlage in ihrem zeitlichen Verlauf aufgezeichnet. Die so entstehende Spur und der aufzuzeichnende Schall stehen dann in einem Verhältnis der Analogie; jede Bewegung des Schalls findet ihre analoge Entsprechung in einer ,Bewegung' der Spur - mit leichten Verlusten. Eben dies ermöglicht es, aus der Spur wiederum Schallwellen zu erzeugen, die analog zur Spur und mittelbar zum ursprünglichen Schall sind - wenn auch wiederum Präzisionsverluste die Reproduktion verfälschen. Durch dieses Verfahren geht allerdings sowohl die Verlustfreiheit der Kopie verloren (denn der kleine Unterschied zwischen Analogaufzeichnung bzw. -reproduktion und Original macht tatsächlich etwas aus) als auch (zumindest zunächst) die Möglichkeit, schon an der aufgezeichneten Spur ihr möglicherweise inhärente digitale Zeichen entziffern zu können. Für Edisons Erfindung liegt genau darin aber ein Gewinn: Die Reproduktion des Schalls wird auf der Basis des reinen Ornaments vorgenommen, ohne dass eine Differenz von Figur und Ornament vorab getroffen werden müsste.

Genau dieser Punkt kann erklären, weshalb die Edison'sche Phonographie Bedeutung für die Semantik der menschlichen Seele erhalten konnte. Der Phonograph verkörperte in isolierter Form ein Vermögen, das man zuvor als nicht abzulösenden Bestandteil des Vermögenskonglomerats der Seele angesehen hatte. Das lässt sich beispielsweise erkennen, wenn man Herders frühe Überlegungen zu Sprache, Kultur und Seele zu Rate zieht. ${ }^{9}$ Herder hat den Begriff der ,Seele' so beschrieben, dass er präzise mit den Begriffspaaren analog/digital und Figur/Ornament in Verbindung gesetzt werden kann. ${ }^{10}$ Die Unterscheidung zwischen Figur und Ornament ist - so meine Behauptung - grundlegend für menschliche Kommunikation überhaupt (und noch etwa der Luhmann'schen Unterscheidung von Mitteilung und Information vorgeschaltet). Zugleich ist sie in ihren Grundlagen kontingent:

9 Sie finden sich etwa in der sogenannten ,Sprachursprungsschrift ${ }^{\prime}(1772)$ und in „Vom Erkennen und Empfinden der menschlichen Seele“ (1774).

10 Vgl. Till Dembeck: X oder U? Herders ,Interkulturalität', in: Dieter Heimböckel/Irmgard Honnef-Becker/Georg Mein/Heinz Sieburg (Hg.): Zwischen Provokation und Usurpation. Interkulturalität als (un)vollendetes Projekt der Literatur-und Sprachwissenschaften, München 2010, S. 127-151. 
Abb. 1: Antique Phonograph Monthly II/5 (May 1974), Titelblatt.

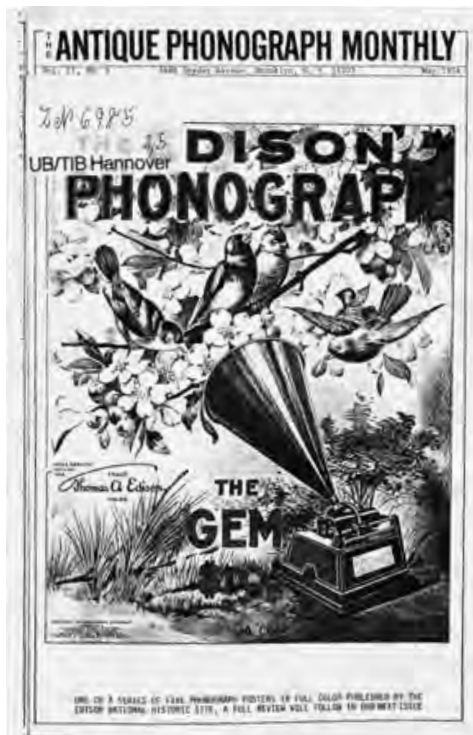

Zwar werden Figur/Ornament-Unterscheidungen stabil immer wieder getroffen, aber es ist nicht vorhersehbar, was an Ornament vielleicht doch in einer bestimmten Situation figurativ wird werden können. Das Vermögen, diese Unterscheidung dennoch so zu treffen, dass sich Sinn ergibt, gilt nun Herder, auch wenn er dies in anderen Worten sagt, nicht nur als kulturstiftend, sondern es bildet seiner Auffassung nach den Grund der menschlichen Seele. Diese Neubeschreibung spitzt den traditionellen Begriff zu: Der Seele wird zum einen ein privilegierter Zugang zum allenfalls analog zu erfassenden Ornament der Sinnesdaten zugeschrieben, für das sie in besonderer Weise empfänglich ist und in dem sie sich am reinsten ausdrückt; sie vermag dieses Ornament aber zum anderen - und dies ist Herders Einsicht zugleich digital aufzufassen bzw. in digitalen Code zu transformieren. Dass sie dies vermag, bleibt dabei (im Grunde: bis heute) ebenso unerklärlich wie das rein Ornamentale dem digitalen Zugriff unzugänglich bleiben muss. Gerade in dieser Rätselhaftigkeit liegt wahrscheinlich der Grund dafür, dass die Seele als Kerneinheit menschlichen Lebens erscheinen kann.

Vor dem Hintergrund dieser Semantik tritt Edisons Phonograph als eine Technologie in Erscheinung, die, insofern sie für das Ornament der Sinnesdaten empfindlich ist, in die der Kulturtätigkeit vorgeschalteten (animalischen) Tiefenschichten der menschlichen Seele vordringt. Wenn von Beginn an die Originaltreue der Schallwiedergabe durch den Phonographen betont wird - der Apparat gebe etwa die menschliche Stimme „with all their original characteristics“ wieder ${ }^{11}-$, so wird

11 Thomas A. Edison: The Phonograph and its Future, in: The North American Review CXXVI/6 (1878), S. 527-536 (hier: S. 530). 


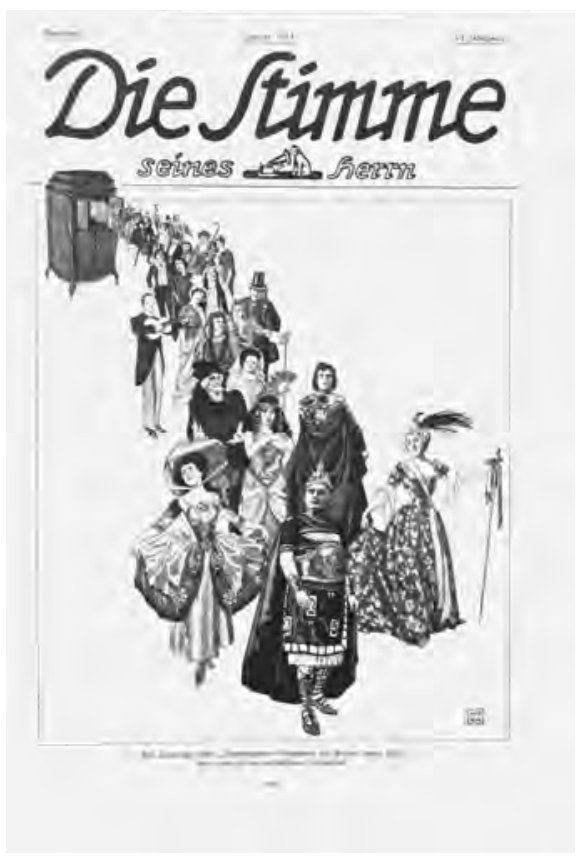

Abb. 2: aus: Die Stimme seines Herrn VI/1 (Januar 1914), Titelblatt.

dabei insbesondere seine Fähigkeit hervorgehoben, menschliche Stimmungen und Gefühlsausdrücke zu reproduzieren, die gerade das Ornament der menschlichen Rede widerspiegelt, die sich sich aber der digitalen Erfassung entziehen. Mit der Aufzeichnung der stimmlichen "intonations, inflections, pauses, and quality “12 macht der Phonograph "the whole world of expression in speech and song "13 reproduzierbar, er ist „prepared to represent all moods“. ${ }^{14}$ Mit dem Phonographen eröffnet sich ein medialer Kanal, der über das Ornament der Stimme einen analogen Zugang zur Tiefenschicht der menschlichen Seele legt. Die Überzeugung, damit auch einen tieferen Bereich des Lebens erschlossen zu haben, zeigt sich in der Vorstellung, der Phonograph täusche selbst die sensorische Wahrnehmung der Tiere er lässt, so suggeriert Edisons Firma in einem Werbeplakat, selbst Singvögel glauben, er sei ihresgleichen [Abbildung 1]. Hiervon ausgehend etablieren sich weitergehende Kontrollphantasien, denen der Rückgriff auf Analogmedien gemein ist: Das Leben und andere (vielleicht auch noch gänzlich unbekannte) Phänomene werden, wie der Schall, als Wellenbewegungen aufgefasst, die ineinander transformierbar

12 Anonym: The Edison Phonograph, in: The Popular Science Monthly XII (April, 1878), S. 748-749 (hier: S. 749).

13 George B. Prescott: The Telephone and the Phonograph, in: Scribner's Monthly XV (April 1878), S. 848-858 (hier: S. 857).

14 Frank Z. Maguire: The Graphophone, in: Harper's Weekly XXX (17.7.1886), S. 458-459 (hier: S. 458). 
sein sollen. Aufgrund dieser Transformierbarkeit erhofft man sich dann, die einen Wellenphänomen mittels anderer kontrollieren, ja, manipulieren zu können. So wie man die Singvögel durch den Phonographen zum Singen bringt, mag man auch die Seele durch die Übertragung analoger Signale auf neuen Kanälen fernsteuern können. ${ }^{15}$

Es ist nur konsequent, wenn mit dem Kontrollgewinn über vormalige Ausdrucksmedien der ,Seele' schließlich sogar Vorstellungen von Unsterblichkeit verbunden werden: „Will it not be like holding communion even with immortality?" 16 So fragt Emile Berliner, der Erfinder des Grammophons, und die Vorstellung, mit einem Phonogramm der eigenen Stimme sich selbst unsterblich machen zu können, ist allgegenwärtig. Man diskutiert ausgiebig die Möglichkeit, mit dem Phonographen auch die Stimmen der Toten wiedergeben zu können - und ein Phonogramm, das angeblich die Aufzeichnung der letzten Worte eines hingerichteten Mörders enthielt, verkaufte sich massenhaft. ${ }^{17}$ Aber der Phonograph erscheint nicht nur als ein Medium, das den Tod überwindet. Die Trennung von Körper und Seele, die der Phonograph vordergründig vollzieht, führt vielmehr folgerichtig sogleich zur serienmäßigen Produktion teillebendiger Phänomene - angefangen mit den sprechenden Puppen, die Edison schon 1890 (mit sehr mäßigem Erfolg) vermarkten ließ. Die neue Ortsunabhängigkeit der Stimme lässt Musiker, später ganze Orchester in den Wohnzimmern erscheinen - die hier, wie eine Illustration aus der Neujahrsausgabe der Zeitschrift Die Stimme seines Herrn von 1914 zeigt, eine gespenstische Zweitexistenz führen [Abbildung 2]. Dass der Eindruck des Gespenstischen entsteht, hat damit zu tun, dass der Phonograph die Toten eben nur unvollständig belebt. Er macht eine Kopie (wenn auch keine verlustfreie!) von Phänomenen, in denen sich die menschliche Seele ausspricht und die bisher auch nur die Seele erfassen konnte. Zugleich aber fehlt ihm das für die menschliche Seele grundlegende Vermögen, zu digitalisieren und damit Sinn zu produzieren. Der Phonograph und die Stimmen, die er reproduziert, sind nur unvollständig lebendig, weil die neue Medientechnik durchschneidet, was zuvor in der Seele zusammenhing: das analoge Empfinden der Seelensprache und den damit einhergehenden Zugang zu tieferen Schichten des Lebens auf der einen und das Vermögen der Seele zur Digitalisierung auf der anderen Seite. Insofern ist der Phonograph von Beginn an ein Medium des Untoten. ${ }^{18}$

15 Vgl. hierzu Robert Stockhammer: The Techno-Magician. Fascination around 1900, in: Jarmila Mildorf/Hans Ulrich Seeber/Martin Windisch (Hg.): Magic, Science, Technology, and Literature, Münster 2006, S. 167-177.

16 Mit dieser Frage endet die bei Frederic William Wile: Emile Berliner. Maker of the Microphone, Indianapolis 1926, S. 172ff., wiedergegebene Rede Berliners vor dem Franklin Institute of Philadelphia vom 16.5.1888.

17 Tom Brooks: The Last Words of Harry Hayward (A True Record Mystery), in: The Antique Phonograph Monthly I.6 (1973), S. 1, 3-9.

18 Dass Untote um 1900 Medienmetaphern sind, sehen bereits Kittler und - in seiner Nachfolge und pointierter - Rickels. Für Kittler leisten die neuen Medien um 1900, also insbesondere Phonograph, Film und Schreibmaschine, die manifeste Unterwerfung oder Subver- 
Das bedeutet auch, dass Edisons Idee, Bücher qua Phonograph hörbar zu machen, zwar einerseits einen Gewinn verspricht; denn jenseits des bloß digitalen Codes des Buchs ließe sich dann durch die Stimme des Vorlesers Einfluss auf das klangliche Ornament nehmen und dem bloßen Text eine Dimension hinzufügen, die der Tradition zufolge eine besonders unmittelbare Verbindung zu den Regungen der Seele unterhält. Andererseits aber hat dieses Supplement seine Tücken, denn die Seele, die der digitale Text so gewinnt, kann immer nur Wiedergänger einer, echten' menschlichen Seele sein.

\section{II}

Von dieser semantischen Gemengelage ausgehend verhandelt Stokers Dracula das Verhältnis zwischen Textualität und Phonographie. Vordergründig handelt der Text von einem Kampf zwischen Gegnern, die unterschiedlicher nicht sein könnten: auf der einen Seite die mit den neuesten Technologien ausgerüsteten Vampirjäger, auf der anderen Seite der untote Dracula selbst, der allenfalls archaische, dafür aber umso übernatürlichere (Medien-)Techniken beherrscht. Der Romantext besteht, so die Herausgeberfiktion, ausschließlich aus Aufzeichnungen, welche die einzelnen Vampirjäger gemacht haben, während sie erste Bekanntschaft mit dem Untoten machen, zu einander finden und schließlich zur systematischen Jagd übergehen. Diese dokumentarische Zusammenstellung trägt entscheidend zum Erfolg der Jagd bei, denn sie ermöglicht es den Jägern, ihre Einzelbeobachtungen zu einem sich vervollständigen Bild des Unwesens zusammenzufügen, das Dracula treibt.

sion des immer nur scheinbar souveränen Subjekts unter anti- oder übersubjektive Medien - und dies selbst dann, wenn sie im Dienste von „Herrendiskurse[n]“ zu stehen scheinen, die das souveräne Ich stützen wollen; Friedrich Kittler: Draculas Vermächtnis (Anm. 1), S. 19. Diese Verknüpfung von Mediengeschichte und psychoanalytischer Theorie präzisiert Laurence A. Rickels: The Vampire Lectures, Minneapolis 1999. Ausgehend von Stokers Roman beschreibt er Vampirismus als Gegenmodell zur ödipalen Enkulturation. Beruht letztere auf dem Erfolg von Trauerarbeit, also auf dem Prinzip der vollständigen Substitution des begehrten, aber unerreichbaren Objekts durch ein anderes (Inzestverbot), so stellt der Vampirismus die Trauer auf Dauer, erlaubt es nicht, das Verstorbene (den unzugänglichen Gegenstand der Begierde) noch einmal (innerlich) zu töten, sondern schließt es, wie Rickels schreibt, in einer ,Gruft' ein (S. 27, 40-43, 54-56). Die neuen (Tele-)Kommunikationsmedien sind nun für Rickels „more vampiric than even vampirism itself“ (S. 38), weil sie dem ödipalen Prinzip der Substitution und Reproduktion das Prinzip der technisch-medialen Replikation entgegenstellen. Diese Replikation „replace[s] vampirism's antiquated recycling system of death and the dead with the live transmission of gadget love, the one we have no trouble listening in because we are living on in it?" (S. 51) So anregend und überzeugend diese Argumentationsgänge sind - sie differenzieren zu wenig zwischen den unterschiedlichen Möglichkeiten von Verzeichnung, die digitale und analoge Medien bieten, um den hier herausgestellten Zusammenhang zwischen der Medienstruktur des Phonographen, der Semantik des Seele und der Konjunktur des Untoten um 1900 sehen zu können. 
Medienwissenschaftliche Lektüren haben die Medientechniken der Vampirjäger, darunter auch ihre Phonographen, oft nur im Gegensatz zu dem unheimlichen Phänomen gesehen, gegen das man sie einsetzt. ${ }^{19}$ Gegen diese Auffassung gilt es, die Verbundenheit gerade des Analogmediums Phonographie mit dem Untoten auch im Roman herauszuarbeiten - und zwar in Abgrenzung von den digitalen neuen Medien, die bei der Jagd eine Rolle spielen, insbesondere der Schreibmaschine. ${ }^{20}$

19 Vgl. zum Beispiel Rosemary Jann: Saved by Science? The Mixed Messages of Stoker's Dracula, in: Texas Studies in Literature and Language, Vol. 31, No. 2: Nineteenth-Century Fiction (Sommer 1989), S. 273-287. Janns Argument richtet sich in erster Linie gegen die bei John L. Greenway: Seward's Folly: Dracula as a critique of ,normal' science, in: Stanford Literature Review 3 (1986), S. 213-230, zu findende Behauptung, Stokers Roman stelle eine Kritik der ,harten' Wissenschaften dar. In dieser Argumentation ist auch die eingesetzte Medientechnik Teil des siegreichen Verbunds von Männlichkeit, Moral und wissenschaftlicher Vernunft, den die Vampirjäger verkörpern.

20 Gerade diese Differenz wird weder von Kittler noch von Rickels hinreichend berücksichtigt, die die ,neuen Medien' eher als einen Verbund ansehen. Kittler charakterisiert den Roman durchaus treffend als „immer wieder verkannte[s] Heldenepos vom Endsieg technischer Medien über Alteuropas blutsaugerische Despoten“; Friedrich A. Kittler: Grammophon Film Typewriter, Berlin 1986, S. 135. Er sieht ihn auch als Darstellung des Kampfs eines „Herrendiskurses, der immer noch die Phantasmen Ich und Welt aufrechterhält", gegen das grenzüberschreitende Andere des weiblichen Begehrens, an das der Vampir appelliert; Ders., Draculas Vermächtnis (Anm. 1), S. 18. Dabei wird nebenbei durchaus deutlich, dass der Phonograph der Gegenseite stärker verbunden ist als die Schreibmaschine (ebd., S. 42f.), aber daraus werden keine Konsequenzen abgeleitet. Rickels' Behauptung, der Verbund der neuen Medien setze ein vampiristisches Replikationsprinzip an die Stelle der ödipalen Reproduktion, das durch den Nachweis gestützt wird, dass sich unter den Vampirjägern eine neue Form der Gruppenkonstitution etabliert (man denke an die multiple Vaterschaft von Mina Harpers Sohn), läuft immerhin auf eine Differenzierung zwischen den unterschiedlichen neuen Medien hinaus. Demzufolge dient die Schreibmaschine als „control panel“, das innerhalb des neuen Medienverbundes das Eindringen des ,echten Untoten durch die Phonographie verhindern soll; Laurence A. Rickels: The Vampire Lectures (Anm. 18), S. 62. Wicke wiederum differenziert gar nicht zwischen den unterschiedlichen Medien, und kommt - bei im einzelnen sehr überzeugenden Beobachtungen - zu dem Ergebnis, gerade in der Schreibmaschine verkörpere sich der spezifische Vampirismus der modernen Konsum- und Medienkultur am reinsten; Jennifer Wicke: Vampiric Typewriting: Dracula and its Media, in: English Literary History 59/2 (1992), S. 467-493. Das ist um so merkwürdiger, als sie andererseits sehr präzise die Analogie herausarbeitet, die zwischen Dracula und dem Phonographen besteht: „The phonographic records Dr. Seward uses are the reproduction of a voice, of a being, without any body needing to be present, just as Dracula can insinuate himself as a voice into the heads of his followers, or call them from afar." (S. 475) Die einzige mir bekannte Arbeit, die den Phonographen konsequent aus dem Medienverbund aussondert und ihn als verbindendes Element zu Dracula ansetzt, ist Michael Heumann: Ghost in the Machine: Sound and Technology in Twentieth-Century Literature, verfügbar auf: http://thelibrary.hauntedink.com/ghostinthemachine/ [eingesehen am 28.11.2010]: „Dracula is the embodiment of a machine, which, rather than acting as a tool for the benefit of ist human agents, proceeds to suck life out of them." (Kapitel II, Abschnitt II) - Auf die beste mir bekannte Studie zu Stokers Roman, die indes nichts zu den Medi- 
Die Vampirjäger sind der Wissenschaft verpflichtet. Zum Ziel gelangen sie insbesondere durch akribische Gedächtnisarbeit, der sich ja auch die dokumentarische Gestalt des Textes selbst verdankt. Man hat es mit einem Gegner zu tun, der Bereiche der Realität kontrollieren kann, die bislang gänzlich unbekannt waren. Das erfordert eine stark gesteigerte Aufmerksamkeit - und stellt eine Herausforderung für das Entzifferungsvermögen der Vampirjäger dar. Es geht darum, alle Spuren des Untoten zu sichern und möglichst nichts allein dem unzuverlässigen Gedächtnis der Protagonisten anzuvertrauen. Eines der Mittel, die dazu eingesetzt werden, ist der Phonograph. Teile des Textes, nämlich das Tagebuch des Psychiaters Dr. Seward ${ }^{21}$ und ein Memorandum des obersten Vampirjägers van Helsing, ${ }^{22}$ sind ursprünglich auf Phonographen aufgezeichnet worden. Dadurch sollen - so steht zu vermuten - die Gedächtniseindrücke gewissermaßen , unmittelbarer ' registriert werden. Zwar erfolgt die Aufzeichnung immer erst nach dem Ereignis; da sich die Protagonisten aber sofort aussprechen, ohne ihre Erinnerung erst zu disponieren und damit vielleicht schon zu verfälschen, könnte die Hoffnung bestehen, dass sie dem Ereignis näher ist. Dieser Unmittelbarkeitsgewinn wird allerdings um den Preis der völligen Unerschlossenheit des jeweiligen ,Textes' erkauft: Als Mina Harker Dr. Seward bittet, eine bestimmte Stelle des phonographischen Tagebuchs vorzuspielen, wird Dr. Seward erst klar, dass ein solcher unmittelbarer Stellenzugriff angesichts des menschenunlesbaren und paratextuell gänzlich unstrukturierten, also jenseits der unlesbaren linearen Spur keinerlei Orientierungshilfen bietenden Mediums zunächst einmal unmöglich ist, ${ }^{23}$ weshalb sich eine typographische Transkription, also eine Überführung ins Digitale, als notwendig erweist. Nur dank dieser Transkription bleiben die entsprechenden Tagebücher dann auch erhalten, als Dracula zu einem späteren Zeitpunkt die Wachswalzen des Phonographen und andere über ihn gesammelten Dokumente in Flammen aufgehen lässt. Dr. Seward berichtet in seinem Tagebuch von Lord Goldalmings Erzählung des Ereignisses: „,...] All the manuscript had been burned, and the blue flames were flickering amongst the white ashes; the cylinders of your phonograph too were thrown on the fire, and the wax helped the flames.' Here I interrupted. ,Thank God there is the other copy in the safe." 24 Die Betonung liegt hier auf dem Wort copy - gemeint ist damit die verlustfreie, digitale Kopie.

Jenseits des Gegensatzes zwischen Wissenschaft und Übersinnlichem ergeben sich jedoch Überschneidungen. Insbesondere ist die Rolle des Phonographen in diesem Text neu zu fassen, denn mit ihm assoziieren sich eben nicht nur Technik und Wissenschaft. Um das zu sehen, bedarf es einer genaueren Charakteristik der beiden Parteien, die der Roman einander gegenüberstellt.

en sagt, sei hier nur verwiesen: Srdjan Smajić: Dracula and Duty, in: Textual Practice 23/1 (2009), S. 49-71.

21 Bram Stoker: Dracula [1897], London u. a. 1994, S. 172, 399.

22 Ebd., S. 374.

23 Ebd., S. $263 f$.

24 Ebd., S. 340. 
Den Untoten kennzeichnet eine Macht, die sich auf einen Bereich jenseits dessen erstreckt, was seine Gegner mit ihrem bisherigen Unterscheidungsvermögen erfassen können. Er besitzt telepathische Fähigkeiten, kann sich Medien entziehen, in denen seine Gegner zwangsläufig erscheinen oder Spuren hinterlassen (Spiegel), er besitzt die Fähigkeit, gerade mittels seiner Stimme, die übrigens zuweilen so wie die des Phonographen als metallisch beschrieben wird, Kontrolle über Tiere und andere Vampire auszuüben. Er legt auch viel Wert auf die präzise Beherrschung der Töne der Fremdsprache Englisch, da er einen Akzent, also die unvollkommene Kontrolle über deren eigentlich bloß ornamentale Dimension, als Machteinschränkung empfände. ${ }^{25}$ Draculas alternativer Wirkungsbereich gründet auf der parasitären Teilhabe am ,eigentlichen' Leben, die normalen Mechanismen des Lebenserhalts sind ihm verschlossen. Er ist aber auch anderen Beschränkungen unterworfen - etwa besitzt er seine besonderen Kräfte nur in der Nacht, sind ihm Knoblauch, Weihwasser und Kreuze ,unberührbar' und bleibt er durch spezielle Techniken verwundbar. Schließlich hat Dracula, wie van Helsing immer wieder betont, nur ein "child-brain “26 man könnte sagen, er hat dasselbe Problem wie Dr. Seward, wenn er versucht, im Phonogramm zu lesen: Er kann die Digitalisierungsleistung, die seine Gegner pausenlos erbringen, nur unvollkommen nachvollziehen; er kontrolliert Welt analog, und seine Seele ist ebenso unvollkommen oder unvollständig wie diejenige des Phonographen.

Seine Gegner haben dem dreierlei entgegenzusetzen: erstens die Fähigkeit zur präzisen Dokumentation aller erhältlichen Daten. Hier setzen die Vampirjäger eindeutig auf Digitalisierung - weshalb es übrigens nur folgerichtig ist, dass van Helsing die ornamentale Seite der Kommunikation, sprich: sein Akzent im Englischen, anders als Dracula offenkundig völlig gleichgültig ist. Zweitens den Rückgriff auf traditionelles Wissen, also auf scheinbaren Aberglauben, der von den Unzulänglichkeiten des Untoten, weiß`. Drittens haben die Vampirjäger durchaus an den telepathischen Kräften des Grafen teil, und zwar mittels der Hypnose, durch die eine zeitweise Verbindung von Mina Harker zu Dracula hergestellt werden kann. Indem sie die Hypnose nutzen und sich auf den Aberglauben verlassen, machen sich die Jäger, ohne in diesen Momenten in irgendeiner Weise auf Wissenschaftlichkeit und Digitalität setzen zu können, von Mechanismen abhängig, die eigentlich dem Wirkungsbereich Draculas angehören. Sie bedienen sich zumindest teilweise analoger Übertragung und Kontrolle - und so erfüllen sich nebenbei Phantasien, wie sie die Semantik der Phonographie um 1900, wie gesehen, tatsächlich hegt.

Vielleicht ist es deshalb auch nur konsequent, wenn am Ende von der Spurensicherung nichts ,Authentisches', wie es in Jonathan Harkers Schlussnote des Textes heißt, bleibt. Wie die wächsernen Phonographenwalzen den Flammen zum Opfer gefallen sind und nur im digitalen Transkript überlebt haben, sind auch die Protagonisten selbst ,durch die Flammen gegangen', wie Harker schreibt:

25 Vgl. Michael Heumann: Ghost in the Machine (Anm. 20).

26 Bram Stoker: Dracula (Anm. 21), S. 360. 


\section{NoTE}

Seven years ago we all went through the flames [...].

[...] It was almost impossible to believe that the things which we had seen with our own eyes and heard with our own ears were living truths. Every trace of all that had been blotted out [...].

[...] I took the papers from the safe where they had been ever since our return so long ago. We were struck with the fact that, in all the mass of material of which the record is composed, there is hardly one authentic document! nothing but a mass of typewriting [...]. We could hardly ask anyone, even did we wish to, to accept these as proofs of so wild a story. ${ }^{27}$

Letztlich verschließen sich die neuen Phänomenbereiche, mit deren Erschließung sich die Vampirjäger so intensiv befasst haben, mit Draculas Tod wieder dem (zumal wissenschaftlichen) Zugriff; die ,Dokumente، verlieren ihren Dokumentcharakter, und dasjenige in den Vampirjägern, was am analogen Herrschaftsbereich des Untoten teilhatte, geht gewissermaßen ebenso in Flammen auf wie zuvor die wächsernen Phonogramme. Nicht zufällig ist Dracula seinerseits immer schon mit dem Wachs assoziiert und wird er gerade im Moment vor seinem Tode als wächsern gekennzeichnet. ${ }^{28}$ Alles, was mit ihm zu tun hatte, ist schlussendlich ganz ausgebrannt.

Von alledem ist auch der Text des Romans selbst betroffen: Denn er ruft einerseits das Phantasma einer Möglichkeit der analogen technischen Kontrolle neuer Phänomenbereiche auf, zieht sich aber andererseits vor ihnen zurück. Die Teilhabe an dem von Dracula beherrschten Bereich ist nur vorübergehend - und muss es offenbar auch sein. Der Text insistiert darauf, ganz im Digitalen bleiben zu wollen - es handelt sich um ein Buch, das die Möglichkeit von sich weist, jenseits des verlustfrei kopierbaren Zeichensatzes, im Bereich des ornamentalen ,Sounds', noch Gestaltungsmöglichkeiten auszunutzen; gewissermaßen also um einen medial konservativen Text, ein Anti-Hörbuch. ${ }^{29}$ Indem er das Phantasma einer Analogkontrolle über Welt und Leben zurückdrängt, lässt er uns letztlich nur eine gewisse Faszination und viel digitalen Text. Protagonisten und Leser werden auf sich selbst zurückverwiesen, auf die geheimnisvolle Fähigkeit der menschlichen Seele zur Generierung verlustfrei kopierbarer Zeichen, die Dracula nur beschränkt beherrscht. Die darin liegende Teilhabe der Seele am sinnlich Ornamentalen wird nicht weiter erforscht. Das neue Untote, das der Phonograph ebenso symbolisiert wie den technischen Fortschritt, wird zum Schweigen gebracht.

Der Text endet insofern seltsam ausweglos: Er liefert kein Konzept für die Integration des Analogmediums in Literatur, lässt aber indirekt auch die menschliche

27 Ebd., S. 449.

28 „He was deathly pale, just like a waxen image [...].“ Ebd., S. 447.

29 Vgl. Michael Heumann: Ghost in the Machine (Anm. 20), Kapitel II, Abschnitt I. 
Fähigkeit, überhaupt zu lesen beziehungsweise zu digitalisieren, als ebenso unergründlich wie unkontrollierbar in Erscheinung treten. Verfahren für den Umgang mit diesem Abgrund dürften für eine Poetik des Hörbuchs von grundlegender Bedeutung sein.

\section{III}

Im Vergleich zu Dracula steht in Villiers de l'Isle-Adams L'Ève future die Phonographie deutlich expliziter im Vordergrund. Erzählt wird von der Erschaffung einer ,Andreide', wie es im Roman heißt, also einer künstlichen Frau, deren Stimme durch zwei Phonographen erzeugt wird, und zwar auf der Grundlage der Stimme der Frau, nach deren Vorbild ein Erfinder namens Edison die Andreide konstruiert. Es geht wirklich darum, eine Stimme ,in all its characteristics', also mit all ihrem Ornament, im Text heißt es: „à des millionièmes de vibrations près “30 zu reproduzieren. Entscheidend ist dabei für Lord Ewald, dem Edison die Andreide baut, die Trennung dieser Stimme von der Seele, der sie ursprünglich zugehört. Gerade ihre höchst vulgäre Seele nämlich lässt Ewald an seiner Geliebten Alicia verzweifeln, und Edison reagiert mit seinem Projekt nur auf einen Wunsch seines Freundes: „Milord Ewald, ne vous êtes-vous pas écrié, tout à l'heure, en parlant d'Elle: ,Qui m'ôtera cette âme de ce corps?' [...] Eh bien! C'EST MOI." ${ }^{31}$

Die Trennung der Stimme und des Körpers der Geliebten von ihrer Seele wird vollbracht, indem der Körper vollständig neu erschaffen wird und die Geliebte sechzig Stunden ,gute Texte auf dem Phonographen einliest: „Voici les deux phonographes d'or, incliné en angle vers la centre de la poitrine [...]. Ils se passent l'un à l'autre les feuilles métalliques de ces causeries harmonieuses - et je devrais dire célestes, - un peu comme les presses d'imprimerie se passent les feuilles à tirer." ${ }^{\text {"32 }}$ Mit diesen sechzig Stunden geistvollen Sprechens soll es auf Jahre hinweg ausreichen, denn ohnehin, so Edisons Argument, verlaufe Kommunikation in der Regel formelhaft; was eine Liebesbeziehung störe, seien die unvorhergesehenen Antworten, die die Erwartungen nur enttäuschen können: „c'est ne que la nouveauté qui

30 Auguste Villiers de l'Isle-Adam: L'Ève Future [1886], hg. von Nadine Satiat, Paris 1992, S. 213. Im Folgenden wird die deutsche Übersetzung des zitierten Textes jeweils in den Fußnoten nach der folgenden Ausgabe aufgeführt: Ders.: Die künftige Eva. Roman, übers. v. Manfred Gsteiger, Zürich 2004. Hier: „auf Millionstel Schwingungen genau“ (S. 173).

31 Auguste Villiers de l'Isle-Adam: L'Éve Future (Anm. 30), S. 169. „,[...] Mylord Ewald, riefen Sie nicht vor kurzem in bezug auf sie: ,Wenn nur jemand diese Seele aus diesem Körper entfernen könnte!' [...] Nun gut! ICH WERDE DAS TUN. “" Ders.: Die künftige Eva (Anm. 30), S. 108.

32 Auguste Villiers de l'Isle-Adam: L'Ève Future (Anm. 30), S. 282. „Hier sehen Sie zwei schief gegen die Brustmitte geneigte Phonographen aus Gold [...]. Sie schieben sich gegenseitig die Metallblätter mit den harmonischen - ich möchte sagen himmlischen - Gesprächen zu, etwa so, wie die Druckerpressen die Abzüge aufeinanderlegen." Ders.: Die künftige Eva (Anm. 30), S. 277. 
nous désenchante." 33 Wenn man demgegenüber nicht nur wünsche, sondern schon wisse, was das Gegenüber sagen wird, könne die eigene Phantasie es bereichern, indem sie seiner Antwort durch die eigene Frage im Vorhinein Bedeutung oder Bedeutsamkeit verleihe: „Il est tant de mots vagues, suggestifs, d'une élasticité intellectuelle si étrange! et dont le charme et la profondeur dépendent, simplement, de ce à quoi ils répondent. "34 Es handelt sich bei der Ėve future, oder bei Hadaly, wie sie im Roman heißt, mithin um eine Vervollkommnung der Edison'schen Phonographenpuppe und zugleich um die Erfüllung seiner Hörbuch-Vision, um ein wandelndes Hörbuch sozusagen - aber eines, das, darin vielleicht Luhmanns Zettelkasten ähnlich, zum Kommunikationspartner wird, also dazu fähig ist, einen zu überraschen, obwohl man es mit allem, was es von sich gibt, selbst gefüllt hat. ${ }^{35}$

Ist es aber tatsächlich so, dass mit der Andreide lediglich eine komplexe neue Medientechnologie entworfen wird, die das Prinzip der analogen Schallreproduktion mit einer geschickten Kombinatorik - also einem digitalen Verfahren - verbindet und den Rest der Einbildungskraft des ,Benutzers' überlässt? ${ }^{36}$ Im Widerspruch zu einer solchen Deutung gibt es viele Hinweise darauf, dass Hadaly auch Dinge sagt, die nicht vorgesprochen sind. Da sind zum einen die allerersten Dialoge, die die Andreide nach ihrer Fertigstellung mit Lord Ewald führt, der sein Kommen sehr unvermittelt angekündigt hat und noch gar nicht weiß, dass er nicht mit der ,echten' Geliebten spricht: „Mais, enfin, mon cher enchanteur, murmura Lord Ewald, comment se fait-il que Miss Hadaly puisse répondre à ce que je lui dis? Il me semble de toute impossibilité qu'un être quelconque ait prévu mes questions, au point, surtout, d'en avoir gravé d'avance les réponses sur de vibrantes feuilles

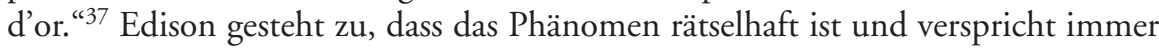
wieder eine vollständige Auflösung - liefert sie aber letztlich nie.

Stattdessen wird das Problem durch viele Informationen, die der Text streut, noch deutlich verschärft. So steht im Hintergrund des Experiments mit der Andreide eine Frau mit dem Namen Sowana, die als Medium fungiert und unter ande-

33 Auguste Villiers de l'Isle-Adam: L'Ève Future (Anm. 30), S. 289. „[N] ur das Neue ist's, das uns ernüchtert." Ders.: Die künftige Eva (Anm. 30), S. 288.

34 Auguste Villiers de l'Isle-Adam: L'Ėve Future (Anm. 30), S. 285. „Es gibt so viele unbestimmte, verfängliche, in ihrem Gehalt sehr merkwürdig schillernde Wörter! Und ihr Reiz, ihre tiefere Bedeutung hängen einfach davon ab, worauf sie antworten!" Ders.: Die künftige Eva (Anm. 30), S. 282.

35 Vgl. Niklas Luhmann: Kommunikation mit Zettelkästen. Ein Erfahrungsbericht, in: Ders.: Universität als Milieu. Kleine Schriften, hg. v. André Kieserling 1992, S. 53-61.

36 Davon scheint Kittler auszugehen, vgl. Friedrich A. Kittler: Aufschreibesysteme (Anm. 1), S. $441 f$.

37 Auguste Villiers de l'Isle-Adam: L'Ėve Future (Anm. 30), S. 238, vgl. auch S. 284. „Aber wie ist denn nun möglich, mein lieber Zauberkünstler', flüsterte Lord Ewald, ,daß Hadaly auf das, was ich ihr sage, zu antworten vermag? Es scheint mir völlig ausgeschlossen, daß ein menschliches Wesen meine Fragen im voraus wissen und erst recht noch die Antworten auf sensible Goldfolien aufzeichnen konnte. [...]““ Ders.: Die künftige Eva (Anm. 30), S. 210, vgl. auch S. 281. 
rem hellseherische Fähigkeiten besitzt. Edison suggeriert, es sei Sowana gelungen, „de pouvoir, á l'occasion, s'Y [in Hadaly] INCORPORER, ELLE-MÊME ET L'AMINER DE SON ÉTAT ,SURNATUREL.“" ${ }^{8}$ Die Phantasie, die sich dahinter verbirgt, ist aus der frühen Semantik des Phonographen bekannt: Das einfache Modell der analogen Schallreproduktion soll zur analogen Kontrolle ,tieferer 'Strukturen des Lebens genutzt werden, ja, das Leben selbst wird als analog kopierbares Schwingungs- oder Wellenphänomen aufgefasst, das sich der digitalen Erfassung entzieht. So wird suggeriert, die Andreide werde mittels eines Fluidums gesteuert: „c'est d'un autre fluide à l'action duquel l'Andréide se trouve soumise en ce moment. Ce fluide, on

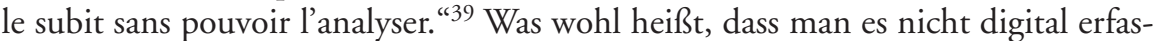
sen kann. Auch Edison und Sowana stehen über ein Fluidum in Verbindung ${ }^{40}$ was die Vermutung nahelegt, eine solche Verbindung könne auch bei der ,Beseelung' Hadalys im Spiel sein. Es ist sogar allgemein die Rede von einer strukturellen Ähnlichkeit von Elektrizität, dem Fluidum und der sogenannten „MATIÈRE RADIANTE " ${ }^{41}$ - offenbar allesamt Phänomene, die so wie Schallwellen und Phonogramm in einem analogen Verhältnis zueinander stehen und laut Edison ebenso leicht technisch zu kontrollieren sein sollen, so dass Telepathie und ,Seelenübertragung' gleichermaßen möglich scheinen.

Schließlich könnte es auch sein, dass die Phantasie Ewalds nicht nur scheinbar, sondern gewissermaßen faktisch an der Belebung Hadalys beteiligt ist. Darauf deuten nicht nur die Hinweise Edisons gegenüber Sowana, es bedürfe einer dritten Person, um Hadaly zu vollenden, ${ }^{42}$ sondern auch die Worte der Andreide selbst. ${ }^{43}$ Hadaly entwirft in einem langen Gespräch mit Ewald eine geradezu romantische Theorie der Imgination. Im Traum, so Hadaly, „['] $]$ homme a conscience [...] de la réalité d'un autre espace inexprimable et dont l'espace apparent, où nous sommes enfermés, n’est que la figure." ${ }^{44}$ Es ist sicher nicht ganz falsch, bei dem Wort ,Figur

38 Auguste Villiers de l'Isle-Adam: L’Ėve Future (Anm. 30), S. 399. Sie habe „,[...] die Möglichkeit besitze[n] “ wollen, „,gelegentlich SICH SELBST IN IHR [Hadaly] ZU VERKÖRPERN UND SIE mit IHREM ,ÜBERnATÜrlichen' Zustand Zu Beseelen.““ Ders.: Die künftige Eva (Anm. 30), S. 458.

39 Auguste Villiers de l'Isle-Adam: L'Ève Future (Anm. 30), S. 323. „,...] [ E]s ist ein [...] Fluidum, dessen Wirkung die Andreide momentan unterworfen ist. Man wird von ihm beeinflußt, aber man kann es nicht analysieren." Ders.: Die künftige Eva (Anm. 30), S. 340.

40 Auguste Villiers de l'Isle-Adam: L'Ėve Future (Anm. 30), S. 397; Ders.: Die künftige Eva (Anm. 30), S. 455.

41 Auguste Villiers de l'Isle-Adam: L'Éve Future (Anm. 30), S. 189, vgl. auch 323. „Strahlenmaterie", Ders.: Die künftige Eva (Anm. 30), S. 137, vgl. auch 340f.

42 Auguste Villiers de l'Isle-Adam: L'Ève Future (Anm. 30), S. 109; Ders,: Die künftige Eva (Anm. 30), S. 22.

43 Auguste Villiers de l'Isle-Adam: L'Ève Future (Anm. 30), S. 381; Ders.: Die künftige Eva (Anm. 30), S. 430.

44 Auguste Villiers de l'Isle-Adam: L'Ève Future (Anm. 30), S. 376; „,...] ist [der] Mensch sich der Realität eines anderen, unaussprechlichen Raums bewußt, dessen bloße Figur der sichtbare Raum ist, in dem wir eingeschlossen sind [...]“; Ders.: Die künftige Eva (Anm. 30), S. 421 . 
auch an phonographische oder andere analoge Spuren zu denken. Diese Spuren verbleiben nun nicht ganz rätselhaft, vielmehr ist es so, dass „dans la voix du vent plaintif; dans le craquement du bois mort d'un meuble ancien" ${ }^{\text {" } 45}$,Boten ' jenes anderen Raums reden: „Et le premier mouvement naturel de l'Âme est de les reconnaître, en et par cette même terreur sainte qui les atteste. "“46 Als eine solche Botin gibt sich Hadaly aus: „Je suis, vers toi, l'envoyée de ces régions sans borne dont l'Homme ne peut entrevoir que les pâles frontières qu'entre certains songes et certains sommeils. “" ${ }^{47}$ Fast im selben Atemzug erteilt sie Ewald, der weiterhin ihr Rätsel zu lösen bestrebt ist, ein Schweigeverbot - er solle nicht mit Edison über ihre Auskünfte sprechen. ${ }^{48}$ Sie scheint sich als Botin einer anderen Wirklichkeitssphäre von ihrem ,Schöpfer ${ }^{`}$ emanzipiert zu haben und fortan ein Eigenleben zu führen ob nun vermittelt durch ,fluide' Korrespondenzen zu Sowana, zu Edison oder zu Ewald selbst. Als Ewald gegenüber Edison kurz darauf zum letzten Mal seinen Einwand vorbringen will, Hadaly könne unmöglich vorprogrammiert sprechen, verstummt er in Gedanken an ihr Schweigegebot. ${ }^{49}$

Ob er Hadaly faktisch belebt oder nicht - diese Frage stellt sich Ewald zum Schluss selbst nicht mehr; sie scheint auch nicht beantwortbar. Ebenso wird die Frage nach der Bedeutung Sowanas für Hadalys Leben im Ungewissen gelassen: Zwar ist zu vermuten, dass Sowana in ungefähr dem Moment gestorben ist, in dem Hadaly sich emanzipiert hat; Hadaly selbst ,verstirbt' aber wiederum, bevor sie aus dem Reiseschlaf, in den sie versetzt worden ist, erwachen - und bevor geprüft werden kann, ob sie auch ohne Sowana ihr Leben fortsetzen könnte. ${ }^{50}$ Hadaly ver-

45 Auguste Villiers de l'Isle-Adam: L'Ève Future (Anm. 30), S. 377; „,[...] in der klagenden Stimme des Winds, im Knacken des trockenen Holzes in einem alten Möbel [...] “"; Ders.: Die künftige Eva (Anm. 30), S. 423.

46 Auguste Villiers de l'Isle-Adam: L'Ėve Future (Anm. 30), S. 377. „,.... Und die erste natürliche Regung unserer Seele besteht darin, mit und dank dem blanken Schrecken, der ihre Gegenwart bezeugt, sie wiederzuerkennen. [...]. “' Ders.: Die künftige Eva (Anm. 30), S. 423.

47 Auguste Villiers de l'Isle-Adam: L'Ėve Future (Anm. 30), S. 380. „,...] Ich komme zu Dir als Abgesandte jener grenzenlosen Regionen, deren blasse Gemarkung der Mensch nur manchmal zwischen Traum und Schlaf wahrnehmen kann. [...]“" Ders.: Die künftige Eva (Anm. 30), S. 428.

48 Auguste Villiers de l'Isle-Adam: L'Ève Future (Anm. 30), S. 392; Ders.: Die künftige Eva (Anm. 30), S. 448.

49 Auguste Villiers de l'Isle-Adam: L'Ève Future (Anm. 30), S. 404; Ders.: Die künftige Eva (Anm. 30), S. 467.

50 Zum offenen Schluss des Romans vgl. das Nachwort von Gsteiger in Auguste Villiers de l'Isle-Adam: Die künftige Eva (Anm. 30), S. 491-507 (hier: S. 506f.). - Die Frage danach, ob Hadaly ,wirklich' belebt wird und ob ihre Rede vorgeschrieben oder ,lebendig' ist, stellt die Krux jeder Interpretation des Textes dar. Noch Gerould meint sie eindeutig beantworten zu können, wenn er Edison zum "director of a theatrum mundi controlled by higher powers" werden lässt; Daniel Gerould, Rez. v. Villiers de l'Isle-Adam: Tomorrow's Eve, übers. v. Robert M. Adams, 1982, in: Science Fiction Studies 11/3 (1984), S. 318-323 (hier: S. 322). Für Schuerewegen stellt gerade der Hinweis auf das Einwirken solcher höhere Mächte den Ausweg dar, den Villiers findet, um der „panique devant les conséquences de sa fascination“ für 
bleibt gewissermaßen in einem untoten Zustand. Vielleicht hat auch sie nur parasitär am Leben partizipiert, um sich zu erhalten, so wie Dracula vom Blut der Lebendigen ,lebt ${ }^{`}$ - immerhin könnte Sowana für sie ihre Lebenssubstanz verausgabt haben. Eine solche Deutung legt auch die Parallelgeschichte von einer nahezu untoten, aber umso verführerischen Tänzerin nahe, deren Opfer der Mann Sowanas geworden ist. Es gibt sogar eine ganz handgreifliche Parallele zwischen Dracula und Hadaly: Sie beide überqueren das Meer in einem Sarg.

Hadalys ,Tod' ist jedoch nicht nur ein Ereignis innerhalb der Geschichte, die der Roman erzählt, und der Roman beschreibt die Erschaffung und die Zerstörung eines wandelnden Hörbuchs nicht nur. Vielmehr hinterlässt Hadalys Untergang Spuren im Erzählmodus selbst. Denn wenn man als Leser schlussendlich nur per Zeitungsbericht und Telegraph von Hadalys Zerstörung erfährt, wird damit ein Darstellungsmodus abgebrochen, der das Erzählen zuvor dem Prinzip einer analogen Direktübertragung angenähert hat: Der größte Teil des Romans ist völlig zeitdeckend erzählt, man hat den Eindruck, einen Mitschnitt der Gespräche zu verfolgen, die im Laufe eines langen Abends in Edisons Labor stattfinden. Im Abbruch der ,Analogübertragung', dank dessen die entscheidende Frage des Textes - was an Hadaly ist Skript, was ist analog generierte Seelensynthese? - demonstrativ unbeantwortet bleibt, kehrt der Text in einen Erzählmodus zurück, der die Annäherung an das neue Medium gar nicht erst mehr sucht, und macht so auch auf seine eigene Verwurzelung in der literarischen Digitalität aufmerksam. Damit steigert er ein selbstreflexives Moment, das ihm auch zuvor bereits innewohnt, zu einer unmissverständlichen Geste. Denn durchgängig wird das gesamte Geschehen des Romans als literarische Projektion ausgewiesen: Die Hauptfiguren sind Antiken - Edison wird als Archimedes, ${ }^{51}$ Alicia als Venus bezeichnet, ${ }^{52}$ und auch Ewalds Züge sind

das Illusionspotential der Edison'schen Erfindungen und die ästhetischen Möglichkeiten, die sie bieten, entgegenzutreten; Franc Schuerewegen: ,Télétechnè fin de siècle: Villiers de l'Isle-Adam et Jules Verne, in: Romantisme 69 (1990), S. 79-88 (hier: S. 83). Neuere Interpretationen betonen, dass Edison die Kontrolle über sein Geschöpf letztlich entgleitet bzw. er mehr erreicht, als er wissenschaftlich erklären kann, vgl. etwa Robert Stockhammer: The Techno-Magician (Anm. 15), S. 172. Butler liest den Text als Darstellung des scheiternden Experiments, Herrschaft über die weibliche Stimme herzustellen: „Sowana’s animation of Hadaly reveals the specter of what still escapes control of the auratic and visual mastery of Edison, and suggests that the liquid, semiotic properties of the (female) voice ultimately cannot be completely controlled." Kristine Butler: The Man of Heightened Faculties: Aurality, Female Voice and Artistic Identity in Villiers de l'Isle-Adam's ,L Eve future', in: Modern Language Studies 36/2 (2007), S. 64-75 (hier: S. 73). Genauer: „Villiers preoccupation is [...] with the failure of the artist to master and aestheticize a voice that is radically detached from his heightened faculties of percaption" (ebd., S. 74).

51 Auguste Villiers de l'Isle-Adam: L'Éve Future (Anm. 30), S. 100; Ders.: Die künftige Eva (Anm. 30), S. 10.

52 Auguste Villiers de l'Isle-Adam: L'Ėve Future (Anm. 30), S. 138; Ders.: Die künftige Eva (Anm. 30), S. 62. 
„d'une régularité grecque “.53 Wichtiger aber noch ist, dass das zeitdeckende Erzählen durchgängig durch literarische Mottos und anspielungsreiche Kapitelüberschriften begleitet wird, die den Text in vielfältige literarische Traditionen einreihen. In seinem gesamten Verlauf simuliert der Text so zwar eine neue Form der quasi-analogen Sprachaufzeichnung, kommentiert dieses Verfahren aber zugleich von der Warte einer älteren Schrifttradition, bis schlussendlich die Simulation abbricht.

Was also wird hier letztlich für Literatur wie für Phonographie entworfen? Das wandelnde Hörbuch Hadaly stellt ein literarisches Medium vor, das durch die Anreicherung der digitalen Texte, mit denen es gefüttert wird, um das Ornament einer lebendigen Stimme und möglicherweise auch durch die analoge Einflussnahme aus anderen Sphären vielleicht verlebendigt wird. Die Offenheit dieses, vielleicht' und die Gebundenheit des Textes selbst an das Digitale stellt der Roman in seinem Schluss ostentativ heraus.

Dennoch lässt der Entwurf der Andreide eine Poetik des Hörbuchs erahnen, und vielleicht sind die phonographischen Phantasien bei Stoker und Villiers de l'Isle-Adam Entwürfe bis heute wirksamer Hörbuchphantasien. Beide Texte eröffnen ein Spannungsfeld zwischen dem digitalen Skript und seiner ornamentalen Anreicherung, zwischen Buch und Hören, wenn man so will. Beide lesen diese Spannung im Hinblick auf neu zu erschließende (oder auch, bei Stoker: möglichst zu verschließende) Zwischenbereiche des Lebens. Das Ergebnis ist in beiden Fällen, dass sich das Phantasma eines ,Hörbuchs' als untote Vision erweist. Ein Hörbuch wäre in beiden Fällen ein Medium, in dem digital codierte Literatur mit einem stimmlichen Ornament versehen wird, das es als defizitäre Schwundstufe menschlichen Lebens erscheinen lässt. Dabei fällt die Einschätzung des ästhetischen Potentials dieser Zusammenführung unterschiedlich aus: Bei Stoker werden die Kräfte der analogen Kontrolle, wie sie der Graf verkörpert, zwar durchaus mit grausiger Faszination betrachtet, letztlich aber geht es um ihren Ausschluss aus der Sphäre der literarischen Medialität selbst, der sie ihrem Wesen nach fremd bleiben und in der sie, wie der Graf im Spiegel, nicht erscheinen können. Villiers hingegen erwägt zumindest - wie ironisch gebrochen auch immer - die Möglichkeiten einer Steigerung der ästhetischen Eindrücklichkeit einer, analogen Literatur'. Auch bei Villiers wird jedoch deutlich, dass die neue Differenz zwischen digitaler und analoger Verzeichnung das Konzept der menschlichen Seele - und ihres literarischen Stellenwerts - medial herausfordert.

53 Auguste Villiers de l'Isle-Adam: L'Ėve Future (Anm. 30), S. 131; „von antikem Maß“ (Auguste Villiers de l'Isle-Adam: Die künftige Eva (Anm. 30), S. 51). Zur Einordnung des Romans in die Tradition der Pygmalionbearbeitungen vgl. Michelle E. Bloom: Pygmalionesque Delusions and Illusions of Movement: Animation from Hoffmann to Truffaut, in: Comparative Literature 52/4 (2000), S. 291-320. 
Den Zusammenhang zwischen der Medienmetapher des Untoten, digitaler Buchund analoger akustischer Medialität, den Dracula und L'Evve future herstellen, aktualisiert Georg Kleins Hörbuch Schlimme schlimme Medien. Es handelt sich um ein ,echtes' Hörbuch auch in dem Sinne, dass es vier Erzählungen des Autors umfasst, die zum Zeitpunkt seines Erscheinens (2007) noch nicht gedruckt vorlagen - sie sind erst später in überarbeiteter Form in den Band Die Logik der Süße (2010) eingegangen. ${ }^{54}$ Auch wenn erkennbar Manuskript verlesen wird, sind die Texte in diesem Sinne ,born analog ${ }^{\text {' }}$ - sie nehmen im Hörbuch ihre erste greifbare Werkgestalt an.

Die Erzählungen wechseln sich mit vier in ,freier ${ }^{\circ}$ Rede vorgetragenen Reflexionen des Autors zu unterschiedlichen Themen ab. Insbesondere ist einer Vampirgeschichte mit dem Titel „Europa erleuchtet" eine Medienreflexion vorgeschaltet. ${ }^{55}$ Klein erläutert hier, die Faszination eines Mediums liege darin, „dass es [im] Rahmen der Geläufigkeit die Überraschung umschließt", so dass zumindest Klein persönlich beispielsweise der Telefonanruf eines Verstorbenen wie die Einlösung eines Versprechens erschiene, welches das Medium immer schon geborgen hat. Klein weist darauf hin, dass auch jenseits dieser persönlichen Erwartung Beschreibungen von Medien gerne eine Parallelisierung von Medientechnik und ,seelischen' Mechanismen vornehmen. Medienthemen durchziehen dann auch die verlesenen Erzählungen: In „Antennen“ geht es um die dämonische Beseelung des Funks; „Shanghai Schicksal" handelt von der Entführung eines Hörbuchmachers, die sich als Medienkunstaktion entpuppt; „Europa erleuchtet“ skizziert eine Medientheorie des europäischen Sendungsbewusstseins und diskutiert damit eine Problematik, die auch die pseudo-ethnologische Erzählung „Crime Tungu“ umtreibt.

„Europa erleuchtet“, die Erzählung, auf die ich meine Überlegungen hier beschränken möchte, wird aus der Sicht des Gehilfen eines Vampirjägers erzählt, der in Prag einen Vampir aufspürt, welcher dem Jägerpaar dann allerdings entkommt. Die Jäger gehören einer Organisation an, die sich weltweit der Vampirjagd widmet, aber wohl alteuropäische Wurzeln hat - das deutet ihre Bezeichnung als ,Heiliger Bund' ebenso an wie der latente Antiamerikanismus des Erzählers. Der Geheimbund operiert offenbar schon seit sehr langer Zeit im Schatten all jener Organisationen, die im Namen des alten und neuen Europa ihre universelle Wahrheit und Moral verbreitet haben - sei es die (katholische) Kirche, sei es das Heilige Römische Reich, seien es die modernen Kolonialstaaten oder neueste Staatenbünde. Auch wenn keinerlei Verbindungen zu diesen Organisationen namhaft gemacht werden - auf der Seite humaner Wahrheit und Güte, wie sie sie vertreten, sehen sich die Vampirjäger nicht minder eindeutig als Draculas Gegner in Stokers Roman. Zugleich aber haben die Jäger an jenem von ihnen bekämpften und diese

54 Georg Klein: Die Logik der Süße. Erzählungen, Reinbek bei Hamburg 2010.

55 Georg Klein: Schlimme schlimme Medien, 2-CD-Set, Köln 2007, CD 1, Track 2; die Erzählung „Europa erleuchtet“ findet sich auf Track 3 bis 5. 
Prinzipien radikal in Frage stellenden Zwischenreich teil: Im Lauf der Erzählung stellt sich heraus, dass der Erzähler selbst ein Untoter ist, der sich in einem langsamen Prozess in einen Nachtpavian verwandelt hat und den nach wie vor alle Spuren des ,Feindes', auch wenn ihn zugleich Angriffslust und ,Gier' überfallen, sympathisch berühren. Von den menschlichen Vampirjägern wiederum wird berichtet, dass sie an der, Überlebenskraft ' der Untoten partizipieren, deren ,Blut ${ }^{`}$ - oder: ,Fluidum' - sie zur Stärkung ihrer Jugendlichkeit verzehren dürfen.

Dieser Plot gewinnt seine Relevanz für Fragen der literarischen Medialität nun nicht nur dadurch, dass der Erzähler durchgängig auf medientechnologische Zusammenhänge verweist - von in Stein geritzten Runen über verschiedene andere Schriftformen, Gesang und Film bis hin zu modernen Beleuchtungskonzepten und zur Mobiltelefonie wird alles bedacht. Vielmehr stellt sich die gesamte Zwischenwelt des Untoten, die der Erzähler selbst bewohnt, als mediale Schattenseite der europäischen Missionstätigkeit dar. Das ist wörtlich zu nehmen, denn bei dem Erzähler handelt es sich um einen der prominentesten Missionare, die das Christentum in die Welt gesandt hat. Im Augenblick des Zusammentreffens des Erzählers mit dem Prager Vampir zeigt sich, dass die beiden Untoten in ihrem ,eigentlichen' Leben Method und Kyrill gewesen sind, jenes historische Brüderpaar aus Byzanz, das wesentlich die Missionierung der Slawen vorangetrieben hat ${ }^{56}$ und darüber hinaus mit der Erfindung einer neuen Medientechnik in die Geschichte eingegangen ist. Method und Kyrill haben im Rahmen ihrer missionarischen Tätigkeit eine Schrift entwickelt (die sogenannte Glagolica), mit der man die Sprachen der slawischen Völker - und die ihnen eigenen, im Lateinischen und Griechischen ungebräuchlichen Phoneme - erfassen konnte. ${ }^{57}$ In den Erinnerungen des Nachtpavians umfasst die Missionstätigkeit allerdings nicht nur die Alphabetisierung der missionierten Völker und die Übertragung der Frohen Botschaft in ihre nunmehr schriftlich digitalisierten Sprachen; sie stellt sich vielmehr als multimedialer Seelenfang im Dienste der christlichen Wahrheit dar. Gerade Kyrill setzt nicht nur seinen Intellekt und seine sprachliche Einfühlungsfähigkeit ein, sondern auch seine sexuelle Ausstrahlung und vor allem seinen bewegenden Gesang. Diese Fähigkeiten ermöglichen ihm sogar noch die Flucht vor den Vampirjägern: Er bewerkstelligt sie nicht nur, indem er die örtliche Helferin der Jäger verführt, die im entscheidenden Moment eingreift, sondern auch dadurch, dass er den Erzähler, seinen Bruder, in

56 Ch. Hannick: Konstantin und Method, in: Lexikon des Mittelalters, Bd. 5, Stuttgart 1999, Sp. 1382-1385.

57 Vgl. Harald Haarmann: Entstehung und Verbreitung von Alphabetschriften, in: Hartmut Günther/Otto Ludwig (Hg.): Schrift und Schriftlichkeit / Writing and Its Use. Ein interdisziplinäres Handbuch internationaler Forschung. An Interdisciplinary Handbook of International Research, Bd. 2, Berlin/New York 2004, S. 329-347 (hier: S. 344); Nikolaos H. Trunte: Словеньскъй языкъ. Ein praktisches Lehrbuch des Kirchenslavischen in 30 Lektionen. Zugleich eine Einführung in die slavische Philologie, Bd. 1: Altkirchenslavisch, München 2005, S. 24-26, 258-260. 
einer toten Sprache dennoch lebendig anspricht, nämlich auf Byzantinisch. Der Jagdinstinkt des ,guten' untoten Affen auf den ,bösen' untoten Vampir wird zuerst durch diese Illusion eines echten Lebens längst verklungener Rede gebremst - und erst danach durch die Entdeckung der Blutsverwandtschaft.

Fasst man die Vampirgeschichte als Allegorie einer Mediengeschichte europäischer Mission auf - also über die christliche Missionstätigkeit hinaus, die sich ohnehin spätestens im 19. Jahrhundert in eine Missionstätigkeit mit universell zivilisatorischem Anspruch verwandelt -, so lässt sich das untote Dasein der beiden Brüder als Allegorie für Techniken der Analogkontrolle lesen, die zwar einerseits der Schriftbindung dieses Sendungsbewusstseins zuwiderlaufen, das sich durch das digital codierte, rein figurative Wort etabliert, ihm andererseits aber als eine Art Supplement überlebensnotwendig zu sein scheinen. Historisch waren Method und Kyrill einerseits Medienrevolutionäre - sie können getrost als frühmittelalterliche Edisons gelten. Andererseits waren sie Grenzgänger in doppelter Hinsicht, einmal zwischen römischem und byzantinischem Christentum, einmal zwischen Christentum und den slawischen Heiden. Kleins Erzählung bringt diese Grenzüberschreitungen mit dem Einsatz von Medientechniken in Verbindung, die sich nicht auf das Digitale der schriftlichen Botschaft reduzieren lassen - und die deren (Trans-)Mission einem Bereich analoger Formbildung überantworten, in den sich auch ,feindliche" Botschaften einnisten können. Zu diesen ,Medientechniken gehören neben erotischer Manipulation und Gesang späterhin vielleicht auch Film und Phonographie - beide haben für Kolonialisierung und Mission um 1900 eine gewisse Rolle gespielt.

Was bedeutet diese fast schon geschichtsphilosophische Aufladung der Erzählung für die Medialität des Hörbuchs selbst? Man kann die Erzählung - und dank der Korrespondenzen zwischen den Texten auch das ganze Hörbuch - als Statement über europäische Literalität lesen, als Hinweis auf die nie abzustellende Abhängigkeit jeder digitalen Verzeichnung von dem allenfalls analog zu erfassenden Ornament, das ihr anhaftet, und damit auch auf die rätselhaft unkontrollierbare Fähigkeit der Seele, zwischen diesen beiden Polen zu vermitteln - eine Fähigkeit, die der alteuropäischen Semantik zufolge geradewegs ihre Anfälligkeit für das ,Böse' impliziert. Damit ordnet sich das Hörbuch in die Semantik ein, die Dracula und L'Evve Future entfalten; es überführt sie aber in eine Art Kulturkritik, wenn sie die Konflikte, welche die beiden älteren Texte umtreiben, im Blick auf,Europa' zu be- und ,erleuchten' sucht, ohne sie überwinden zu wollen oder zu können. Die Behutsamkeit dieser Kritik nimmt die Medienform des Hörbuchs selbst insofern auf, als sie eine angesichts der technischen Möglichkeiten geradezu vorsichtige Zusammenführung digitaler und analoger Verzeichnungsverfahren anstrebt: durch die Inszenierung unterschiedlicher Sprechweisen, denn im Wechsel zwischen ,freier' und vorlesender Rede inszenieren sich nicht zuletzt unterschiedliche Grade der ,Lebendigkeit' von Sprache; durch die äußerst gedämpfte oder dezente Nutzung von Soundeffekten - in „Europa erleuchtet“ ist als einzigem Lesestück ein sehr leichter Hall zu spüren; durch lautmalerische Elemente, die in der Buchfassung teils nicht mehr auftauchen - dort ist zum Beispiel nicht von der „Tücke des päpst- 
lichen Päderasten“, sondern nur noch von der „Tücke des Papstes“58 die Rede. Kleins Hörbuch wird so zu einem Ort, an dem die unterschiedlichen Arten und Weisen (analog-)medieninduzierter Seelentechnologie miteinander ins Gespräch gebracht werden.

58 Georg Klein: Die Logik der Süße (Anm. 54), S. 230. 


\section{ABBILDUNGSVERZEICHNIS}

Heinz Hiebler: Problemfeld ,HÖRbuCH ${ }^{\star}$

Авв. 1-12: Johann Wolfgang von Goethe: Der Totentanz. Tröger-Mooslechner-Productions, Animation: Steffen Troeger und Andreas Mooslechner, GoLeGo-Animation 2004.

Unter: www.golego.de und http://www.youtube.com/watch?v=2qJ7VYkSZlw [Datum: 30.8.2010].

Авв. 13: Dik Browne: Hägar: Ohne Titel [„Verdammt! Er tut's schon wieder!"]. King Features Syndicate, Inc. 1977, München: Goldmann o. J.

Aвв. 14: Ulrich Boner: Der Edelstein, gedruckt von Albrecht Pfister, Bamberg 1461. [Aus: Stephan Füssel: Gutenberg und seine Wirkung, Darmstadt: Wissenschaftliche Buchgesellschaft 1999, Abb. 42.]

Авв. 15: Ulrich Boner: Der Edelstein, hg. v. Franz Pfeiffer, Leipzig: Göschen 1844, S. 4.

Aвв. 16: Heinz Hiebler: Medienkulturgeschichte im tabellarischen Überblick [@ Heinz Hiebler].

Авв. 17: Heinz Hiebler: Matrix zum Hörbuch von Danielewski/Böhm Das Haus (2009/10) [@ Heinz Hiebler].

Авв. 18-21: Mark Z. Danielewski, Das Haus - House of Leaves, Hörspiel-Bearbeitung von Thomas Böhm, Berlin: Der Audio Verlag 2010. [Screenshots von der DVD.]

Wolfgang Hagen: SynÄSTHESIE Des HÖRbuCHS

Авв. 1: Luigi Russolo: La Musica (1911-12). Estorick Collection, London, UK/ The Bridgeman Art Library. [Aus: Chessa, Luciano: Luigi Russolo, Futurist, Noise, Visual Arts and the Occult, Berkeley: University of California Press 2012, S. 83].

Till Dembeck: Akustische Untote

Aвв. 1: Antique Phonograph Monthly II.5 (May 1974), Titelblatt.

AвB. 2: Die Stimme seines Herrn VI.1 (1914), Titelblatt.

\section{Uwe Wirth: AkUstische ParateXtualität}

Aвв. 1: Druckfehlerverzeichnis, E. T. A. Hoffmann: Lebens-Ansichten des Katers Murr nebst fragmentarischer Biographie des Kapellmeisters Johannes Kreisler in zufälligen Makulaturblättern (1820-1821), in: Ders.: Sämtliche Werke, Bd. 5, hg. v. Hartmut Steinecke/Gerhard Allroggen, Frankfurt am Main 1992, S. 13. 